\title{
An overview of APECOSM, a spatialized mass balanced "Apex Predators ECOSystem Model" to study physiologically structured tuna population dynamics in their ecosystem
}

\author{
Olivier Maury ${ }^{\mathrm{a}, \text { * }}$ \\ ${ }^{a}$ IRD (Institut de Recherche pour le Développement) - CRHMT, av. Jean Monnet, B.P. 171, 34203 \\ Sète cedex, France \\ * Corresponding author : Olivier Maury, Tel.: +33 4995732 28, email address : maury@ird.fr
}

\begin{abstract}
:
This paper gives an overview of the ecosystem model APECOSM (Apex Predators ECOSystem Model) which is developed in the framework of the GLOBEC-CLIOTOP Programme. APECOSM represents the flow of energy through the ecosystem with a size-resolved structure in both space and time. The uptake and use of energy for growth, maintenance and reproduction by the organisms are modelled according to the DEB (dynamic energy budget) theory (Kooijmann, 2000) and the sizestructured nature of predation is explicit. The pelagic community is divided into epipelagic and mesopelagic groups, the latter being subdivided into vertically migrant and non-migrant species. The model is mass-conservative. Energy is provided as the basis of the model through primary production and transferred through 3D spatially explicit size-spectra. Focus species (tunas at present, but any predator species can be considered) are "extracted" from the global size-spectra without losing mass balance and represented with more physiological and behavioural details. The forcing effects of temperature, currents, light, oxygen, primary production and fishing are explicitly taken into account.
\end{abstract}




\section{Introduction}

Present marine ecosystems are experiencing simultaneously the effects of high and increasing levels of fishing pressure (Sinclair and Valdimarsson, 2003) and in depth structural modifications induced by climate change (Hsieh et al., 2005, 2006). The unprecedented speed and magnitude of those global phenomena raise serious concerns about the very fate of marine ecosystems and their potential future (in-)ability to sustain services to human societies such as fisheries or carbon uptake and export. To answer quickly those weighty questions, it is urgent for scientists to achieve a reliable predictive capability through the development of the next generation of ecosystem models which has to embody a high degree of mechanistic details, ecological realism and theoretical consistency. In this perspective, the ecosystem model APECOSM (Apex Predators ECOSystem Model, Maury et al., $2007 \mathrm{a}$ and b) is a recent spatially explicit size-based model of open-ocean ecosystems developed in the framework of the GLOBEC-CLIOTOP synthesis and modelling Working Group (WG4). APECOSM aims at representing the joint effects of environmental variability and fishing on the structure and dynamics of pelagic ecosystems.

APECOSM uses a size-based approach as a practical way to obtain a realistic representation of both the non target organisms (components called OOPC for Open Ocean Pelagic Communities) and the focus species while keeping the functional complexity limited (Fig. 1). In this way, the concept constitutes a concrete implementation of the rhomboid approach to model marine ecosystems as described by De Young et al (2004). APECOSM integrates various physiological, behavioural and ecological processes acting on a variety of scales. It represents the basin wide spatialized dynamics of open ocean pelagic ecosystems from phytoplankton production up to fishing with a special emphasis on predators.

APECOSM is mass conservative. It adopts a process based approach where parameterizations are derived mechanistically and where parameters are biological, physiological or behavioural meaningful constants. Physical forcings (temperature and current), biogeochemical forcings (primary and secondary production, light and oxygen fields) as well as the effects of fishing are explicitely taken into account and constrain the dynamics at various levels.

One of the main characteristic of APECOSM is that the particular structure of regional ecosystems are not prescribed a priori. On the contrary, they are supposed to emerge from the interactions between the global generic structure and set of rules of the model and the regional environmental forcings.

\section{The Open Ocean Pelagic Communities (OOPC)}

The epipelagic OOPC is distinguished from the mesopelagic migratory OOPC which experiences nyctemeral movements and from the mesopelagic non migratory OOPC. The three OOPCs are not taxonomically resolved, their vertical distribution and movements are constrained by light, temperature, food and oxygen (Fig. 2).

Energy fluxes through the ecosystem are controlled by predation. Through size-based predation, 3D fields of primary (flagellates and diatoms) and secondary (micro-zooplankton and meso-zooplankton) production simulated by the biogeochemical model PISCES (Aumont et al., 2003; Aumont and Bopp, 2006) fuel the entire size-spectra of the OOPCs (Fig. 3). Since all the energy fluxes including controlled losses (non predatory mortality, loss of male sexual products and imperfect efficiency of the assimilation process) and dissipation processes (maintenance expenditures and the energetic cost of growth) are explicit, the model conserves energy: predation is a net loss of energy for preys and a net gain for predators. 
Predation is opportunistic and controlled by the ratio of size between organisms through a selectivity function (Maury et al., 2007a) and by the 3D spatial co-occurrence of predator and prey. Growth is a conversion of ingested energy into assimilated energy diminished by a maintenance cost proportional to body weight and then converted into structural material at a given cost (Kooijman, 2000). Reproduction is continuous in time. The model represents energy flux through the OOPCs with size-structured PDEs in 4 explicit dimensions (2D space, time and weight) which combine spatial diffusion and advection terms (accounting for both passive and active movements) with an advection term along the weight dimension representing the growth process and three sink terms for predatory, non predatory and starvation mortality processes. The vertical dimension $z$ is explicitly taken into account through a specific parameterization which integrates vertical advection and diffusion.

$$
\begin{aligned}
\partial_{t} \xi_{t, x, y, w}^{i} & =\operatorname{div}\left(D_{t, x, y, w}^{i} \nabla \xi_{t, x, y, w}^{i}\right)-\operatorname{div}\left(V_{t, x, y, w}^{i} \xi_{t, x, y, w}^{i}\right) \\
& -\partial_{w}\left(\gamma_{t, x, y, w}^{i} \xi_{t, x, y, w}^{i}\right)+\frac{\gamma_{t, x, y, w}^{i} \xi_{t, x, y, w}^{i}}{w} \\
& \left.\left.-\left(\lambda_{t, x, y, w}^{i}+Z_{t, x, y, w}^{i}+M_{t, x, y, w}^{i}\right)_{t, x, y, w}^{i} \quad \text { in } \Omega \times\right]_{e g g}, w_{\max }\right] \times(0, T)
\end{aligned}
$$

Where $\nabla$ and div are the usual differential operators on the spatial domain $\Omega$.

This equation is completed with Dirichlet boundary conditions:

$$
\gamma_{t, x, y, w_{e g g}} \xi_{t, x, y, w_{e g g}}^{i}=R_{t, x, y}, \quad \forall(x, y, t) \in \Omega \times[0, T]
$$

Where $\xi$ is the energy density of the community $i$ of the OOPC, $D$ is the spatial diffusivity matrix, $V$ is the vector of the spatial advection coefficients, $\gamma$ is the growth rate, $\lambda$ is the mortality rate due to predation, $Z$ is the loss of energy from the system due to non predatory mortality and $M$ is the starvation mortality rate. The input of eggs $R_{t}$ into the system due to reproduction is taken into account through the Dirichlet boundary condition in $w=w_{\text {egg }}$.

To ensure a rigorous mass balance, all the coefficients of equations (1) are derived according to the principle of energy and mass conservation (Maury et al., 2007a). The propagation of the variability of primary production through the three OOPCs spatialized sizespectra reflects the complex spatial interplay of bottom-up and top-down processes as well as the 3D effects of ocean temperature, currents, light and oxygen (Fig. 4).

\section{Focus species: Tuna Populations}

APECOSM integrates in a bioenergetic framework the demography of 4 tuna populations (bigeye, skipjack, yellowfin and albacore) with their physiology and main life history traits (growth, reproduction, metabolism, ageing, maturity), their horizontal movements and migrations as well as their small scale vertical movements. As for OOPC communities, the size-structured nature of predation is explicit. The tuna species interact trophically with the three communities of OOPC and are represented with DEB-based (Dynamic Energy Budget, cf. Fig. 5) physiologicaly structured advection-diffusion equations which transport individuals through a 6 dimensions space (2D space, time, reserve, structure, gonad). Length of tunas is proportional to the energy content of structures at the power $1 / 3$ so that the tuna component of the model is fully size structured and hence compatible with the OOPCs. The parameterization of horizontal movements links together advection and diffusion and is derived from a model of individual movements (Faugeras and Maury, 2007). The vertical 
distribution of tunas is explicitly taken into account through a specific parameterization derived from a vertical movements sub-model (Maury, 2004).

$$
\begin{aligned}
& \partial_{t} \zeta_{t, x, y, e, v, g}^{i}=\operatorname{div}\left(D_{t, x, y, v, g}^{i} \nabla \zeta_{t, x, y, e, v, g}^{i}\right)-\operatorname{div}\left(V_{t, x, y, v, g}^{i} \zeta_{t, x, y, e, v, g}^{i}\right) \\
& -\partial_{e}\left(\left(\&_{A}-\psi_{t}\right)_{t, x, y, e, v, g}^{i} \zeta_{t, x, y, e, v, g}^{i}\right)+\frac{\left(\&_{A}-H_{t, x, y, e, v} \zeta_{t, x, y, e, v, g}^{i}\right.}{e+v+g} \\
& -\partial_{v}\left(\left(\mathbb{H}_{\xi}\right)_{t, x, y, e, v, g}^{i} \zeta_{t, x, y, e, v, g}^{i}\right)+\frac{\left(\&_{G}\right)_{t, x, y, e, v, g}^{i} \zeta_{t, x, y, e, v, g}^{i}}{e+v+g} \\
& -\partial_{g}\left(\left(\&_{R}\right)_{t, x, y, y, v, g}^{i} \zeta_{t, x, y, e, v, g}^{i}\right)+\frac{\left(\beta_{R}\right)_{t, x, y, e, v, g}^{i} \zeta_{t, x, y, e, v, g}^{i}}{e+v+g} \\
& -\left(\lambda_{t, x, y, v}^{i}+F_{t, x, y, v}^{i}+Z_{t, x, y, e, v, g}^{i}+M_{t, x, y, e, v, g}^{i}\right) \zeta_{t, x, y, e, v, g}^{i} \\
& \text { in } \left.\left.\left.\Omega \times] 0, E] \times V_{\text {egg }}, V_{\max }\right] \times\right] 0, G\right] \times(0, T)
\end{aligned}
$$

With $\dot{\zeta}$ the energy density of tunas species $i, D$ the spatial $(x, y)$ diffusivity matrix, $V$ the vector of spatial $(x, y)$ advection coefficients, $e, v$ and $g$ the energy density of reserves, structure and gonads, \&, \& \& and \& the rates of energy flux for assimilation, reserve use, structural growth, gonads development, $\lambda$ the predatory mortality, $F$ the fishing mortality, $Z$ the ageing mortality, and $M$ the non predatory mortality. Reproduction is taken into account in the boundary condition in $v$.

Behaviour of fish is related to their physiological status so that physiology acts as a transfer function between environmental variability and behaviour. In APECOSM, tunas behave in order to maximize the number of eggs spawned (growth of adults), and the larval survival (location of spawning). A behavioural shift occurs before spawning, when gonads energy content reaches a fixed level, from movements devoted to growth maximization (to feeding areas) to movements devoted to larval survival (to spawning areas). The model outputs the distribution of tunas which can be compared to observed commercial catches. Preliminary retrospective runs of APECOSM have been conducted in the Indian Ocean, forced by a climatological environment (Fig. 6).

\section{Conclusion}

APECOSM produces a large range of outputs which can be compared with observed data such as size-structured prey fields, fishery catches and size-frequencies, tag-recovery data and archival tagging experiments, size-structured stomach contents, $\mathrm{N}^{15}$ ratios of tunas and of the ecosystem, growth and maturity schedules, etc... Various data assimilation experiments for estimating APECOSM key parameters are currently being undertaken.

In the framework of CLIOTOP WG4, various hindcast and forecast simulation studies will be conducted to address the joint effects of fishing and climate variability on oceanic ecosystems and the tuna community which inhabit them. 


\section{Acknowledgements}

OM would like to thank IGBP programs IMBER and GLOBEC, as well as the Network of Excellence EUR-OCEANS of the European Union's 6th Framework Program for funding the symposium on "Parameterization of Trophic Interactions in Ecosystem Modelling", Cadiz, March 2007, and the meeting conveners for the invitation to participate. This study was part of the REMIGE project funded by the French National Research Agency ANR Biodiversité.

\section{References}

Aumont, O., L. Bopp (2006), Globalizing results from ocean in situ iron fertilization studies, Global Biogeochem. Cycles, 20 (2), 10.1029/2005GB002591.

Aumont, O.; Maier-Reimer, E.; Blain, S.; Monfray, P. (2003). An ecosystem model of the global ocean including Fe, Si, P colimitations Global Biogeochemical Cycles, 17(2), DOI 10.1029/2001GB001745

Faugeras B., O. Maury, 2007. Modelling fish population movements: from an individualbased representation to an advection-diffusion equation. Journal of Theoretical Biology 247: 837-848.

Hsieh, C.-h., S. M., Glaser (2005), Distinguishing random environmental fluctuations from ecological catastrophes for the North Pacific Ocean, 435(7040): 336-340.

Hsieh, C.-h., C. S. Reiss (2006), Fishing elevates variability in the abundance of exploited species, Nature 443: 859-862.

Kooijmann, S.A.L.M. (2000), Dynamic Energy Mass Budgets in Biological Systems, Cambridge University Press, Cambridge.

Maury, O., Y-J. Shin, B. Faugeras, T. Ben Ari, and F. Marsac, 2007b. Modelling environmental effects on the size-structured energy flow through marine ecosystems. Part 2: simulations. Progress in Oceanography. 74(4) : 500-514.

Maury, O., B. Faugeras, Y-J. Shin, J.C. Poggiale, T. Ben Ari, and F. Marsac, 2007a. Modelling environmental effects on the size-structured energy flow through marine ecosystems. Part 1: the model. Progress in Oceanography 74(4) : 479-499.

Maury O., 2004. How to model the size-dependent vertical behaviour of bigeye tuna in its environment? Col. Vol. Sci. Pap. ICCAT, 57(2) (2005) : 115-126.

Sinclair M., G. Valdimarsson, 2003. Responsible Fisheries in the Marine Ecosystem. Sinclair M., G. Valdimarsson (eds), FAO/CABI 2003. 426p.

de Young B., M. Heath, F. Werner, F. Chai, B. Megrey, P. Monfray. Challenges of Modeling Ocean Basin Ecosystems. Science vol 304-4 June 2004, 1463-1466. 


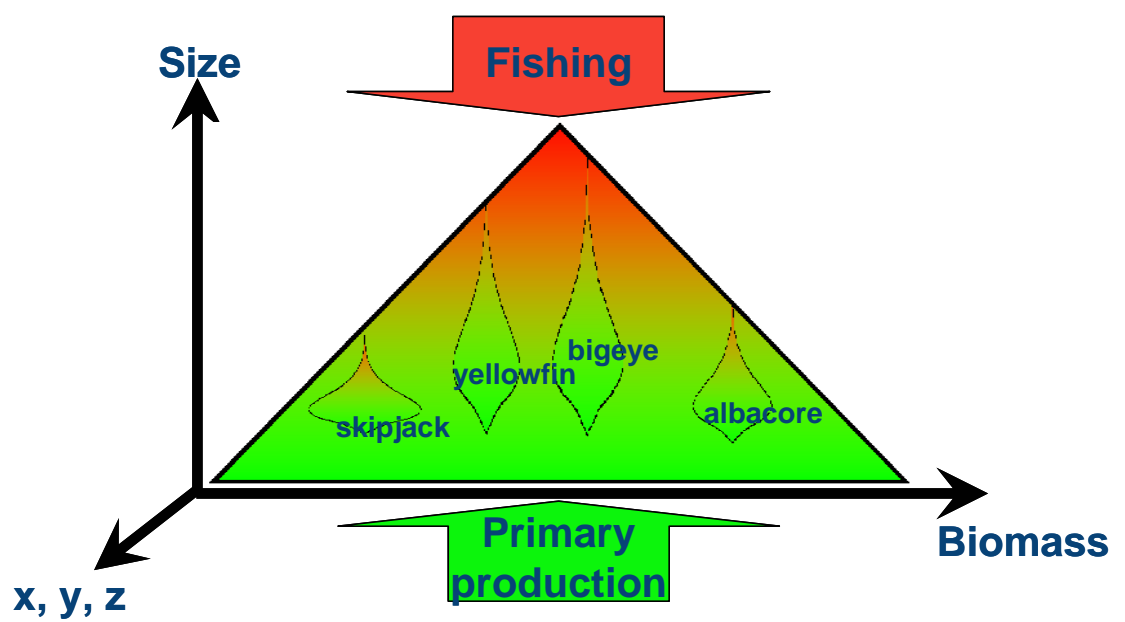

Fig. 1.: APECOSM is a basin scale ecosystem model integrating bottom-up and top-down processes. It represents the overall energy fluxes through the ecosystem with a size spectrum approach. Focus species (tropical tunas) are extracted from this energy flux and represented with more physiological and behavioural details.

Day

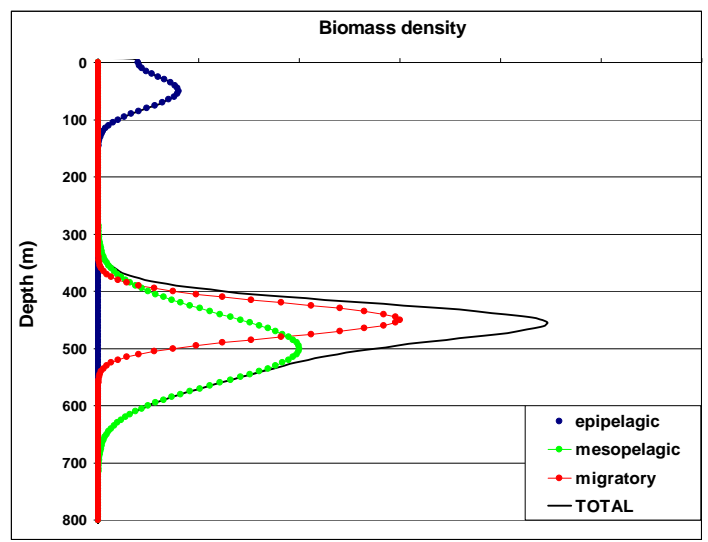

Night

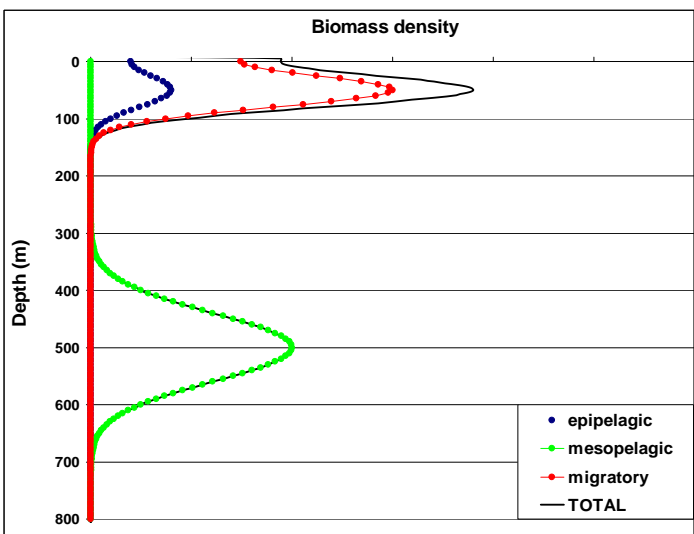

Fig. 2.: Schematic representation of the vertical distribution of the three OOPC communities and their diel vertical migrations. 


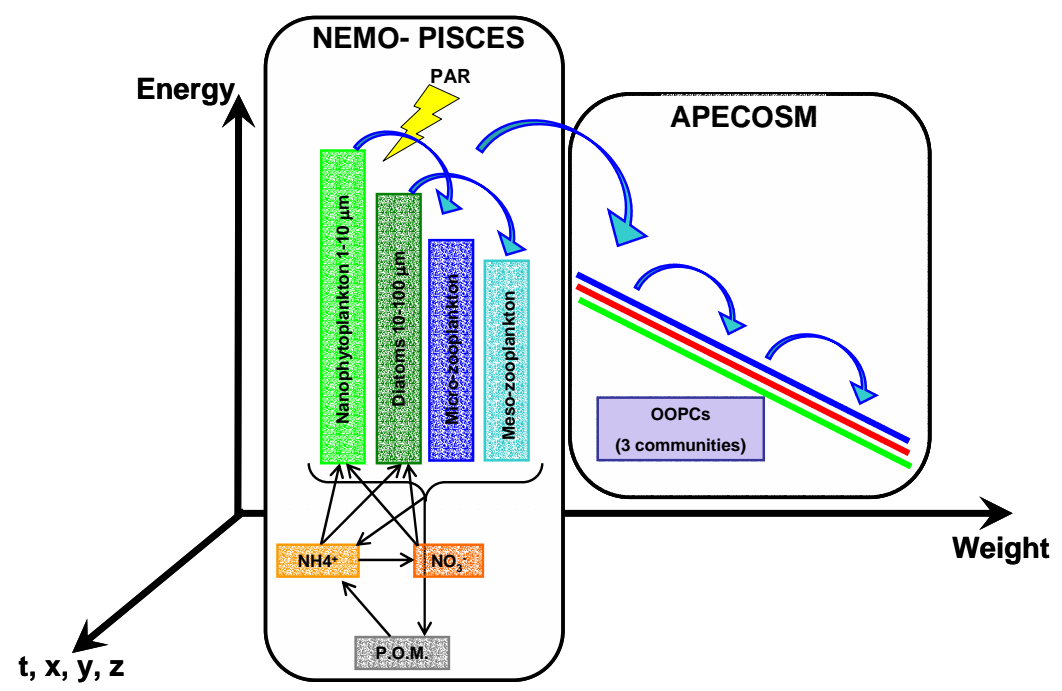

Fig. 3.: Schematic representation of the size-structured energy fluxes between the biogeochemical PISCES (Aumont et al., 2003; Aumont and Bopp, 2006) and APECOSM.

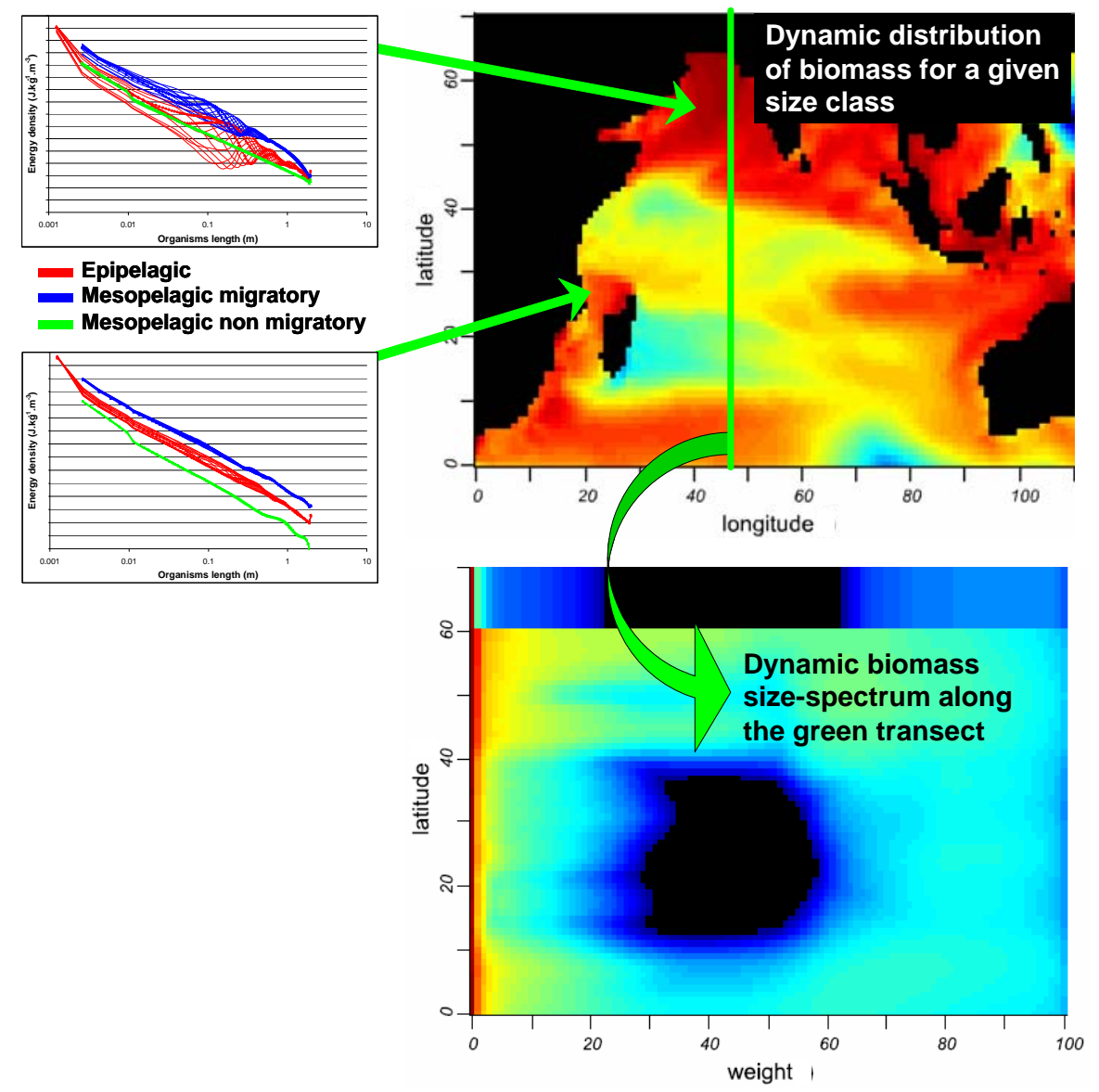

Fig. 4.: Left: size spectra of the epipelagic, mesopelagic migratory and mesopelagic non migratory OOPCs in two regions (top: Arabian Sea ; bottom: Mozambique Channel). A line is drawn for each climatological month. Right top: spatial distribution of the OOPC biomass integrated from $10^{-3} \mathrm{~m}$ to $0.2 \mathrm{~m}$ and cumulated over the three communities in January. Right bottom: spatialized size-spectrum along the green latitudinal transect. 


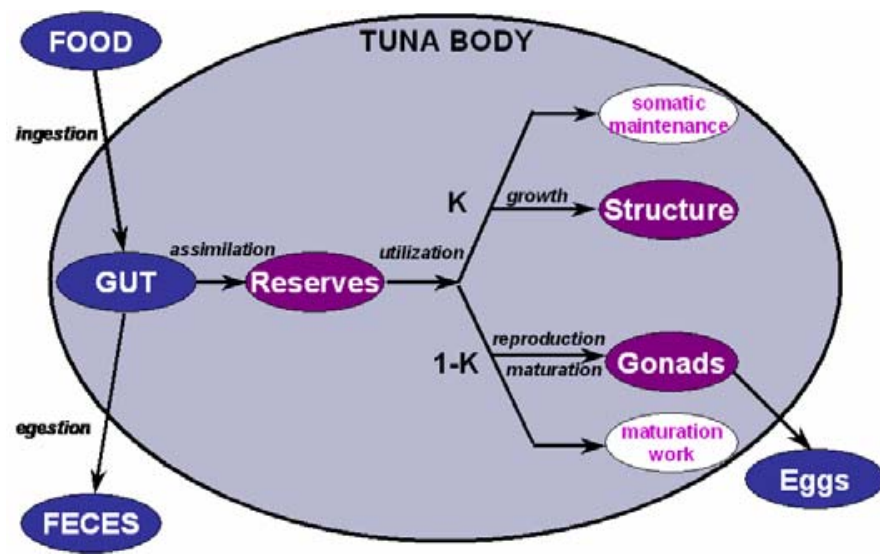

Fig. 5.: Schematic representation of the DEB-based (Kooijman, 2000) physiological structure used in APECOSM. The amount of energy ingested by a predator is proportional to a body surface and assimilated at a given cost. A fraction $\kappa$ is used for somatic growth and maintenance whereas a fraction $(1-\kappa)$ is allocated to egg production and gonadic maintenance. The energetic cost of growth is explicitly considered in the balance equations and the eggs produced are injected at the basis of the population size-range.

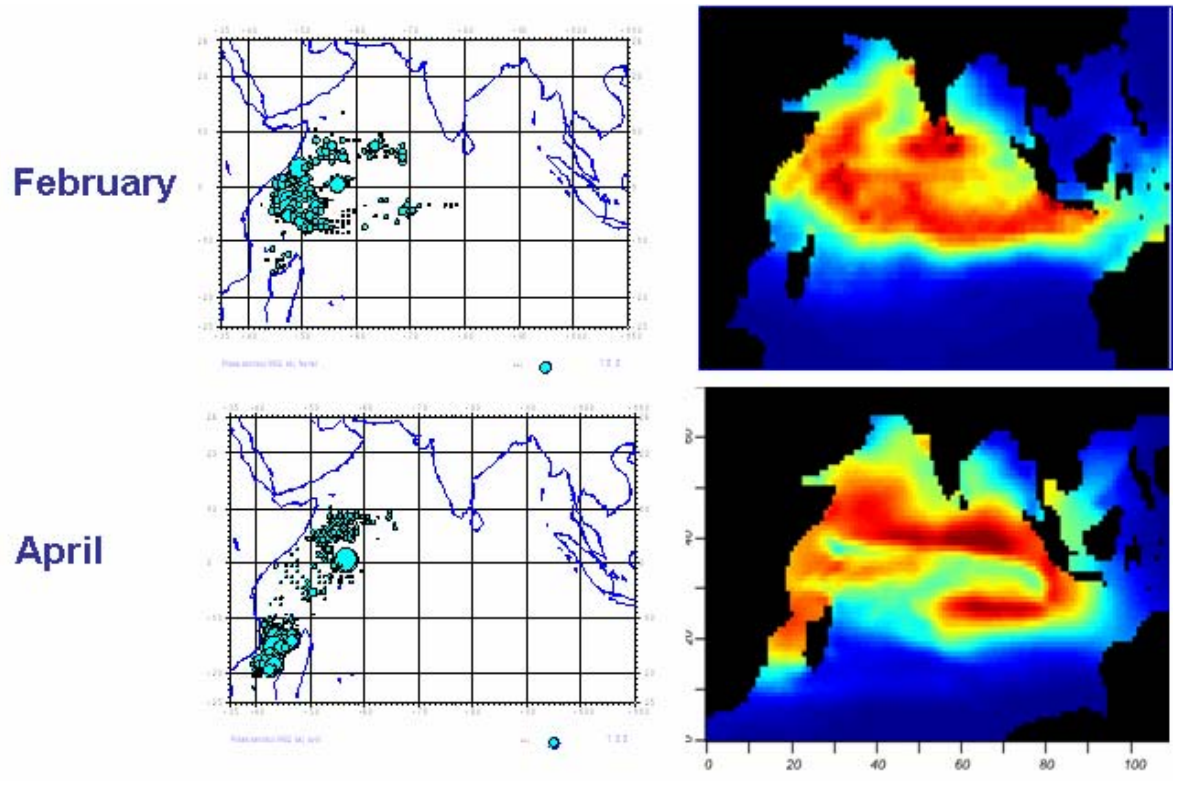

Fig. 6.: Comparison between observed catches of skipjack tuna (Katsuwonus pelamis) in the Indian Ocean (left) with the biomass of skipjack simulated by APECOSM and cumulated over the range of sizes fished (right). 